\title{
Truncation of POC1A associated with short stature and extreme insulin resistance
}

\author{
Jian-Hua Chen ${ }^{1,2}$, Maria Segni ${ }^{3}$, Felicity Payne ${ }^{4}$, Isabel Huang-Doran ${ }^{1,2}$, \\ Alison Sleigh ${ }^{5,6}$, Claire Adams ${ }^{1,2}$, UK10K Consortium*, David B Savage ${ }^{1,2}$, \\ Stephen O’Rahilly ${ }^{1,2}$, Robert K Semple ${ }^{1,2}$ and Inês Barroso ${ }^{1,2,4}$
}

${ }^{1}$ The University of Cambridge Metabolic Research Laboratories, Wellcome Trust-MRC Institute of Metabolic Science, Cambridge, UK

${ }^{2}$ The National Institute for Health Research Cambridge Biomedical Research Centre, Cambridge, UK

${ }^{3}$ Department of Pediatrics, Sapienza University, Rome, Italy

${ }^{4}$ Metabolic Disease Group, Wellcome Trust Sanger Institute, Cambridge, UK

${ }^{5}$ Wolfson Brain Imaging Centre, University of Cambridge, Cambridge, UK

${ }^{6}$ National Institute for Health Research/Wellcome Trust Clinical Research Facility, Cambridge, UK

*The full list of UK10K Consortium members is presented in the Acknowledgements section

Correspondence should be addressed to R K Semple or I Barroso Emails

rks16@cam.ac.uk or ib1@sanger.ac.uk

\begin{abstract}
We describe a female proband with primordial dwarfism, skeletal dysplasia, facial dysmorphism, extreme dyslipidaemic insulin resistance and fatty liver associated with a novel homozygous frameshift mutation in POC1A, predicted to affect two of the three protein products of the gene. POC1A encodes a protein associated with centrioles throughout the cell cycle and implicated in both mitotic spindle and primary ciliary function. Three homozygous mutations affecting all isoforms of POC1A have recently been implicated in a similar syndrome of primordial dwarfism, although no detailed metabolic phenotypes were described. Primary cells from the proband we describe exhibited increased centrosome amplification and multipolar spindle formation during mitosis, but showed normal DNA content, arguing against mitotic skipping, cleavage failure or cell fusion. Despite evidence of increased DNA damage in cells with supernumerary centrosomes, no aneuploidy was detected. Extensive centrosome clustering both at mitotic spindles and in primary cilia mitigated the consequences of centrosome amplification, and primary ciliary formation was normal. Although further metabolic studies of patients with POC1A mutations are warranted, we suggest that POC1A may be added to ALMS1 and PCNT as examples of centrosomal or pericentriolar proteins whose dysfunction leads to extreme dyslipidaemic insulin resistance. Further investigation of links between these molecular defects and adipose tissue dysfunction is likely to yield insights into mechanisms of adipose tissue maintenance and regeneration that are critical to metabolic health.
\end{abstract}

$\begin{aligned} & \text { Key Words } \\ & \text { - POC1A } \text { centrosome } \\ & \text { - centriole } \\ & \text { - primary cilium } \\ & \text { - short stature } \\ & \text { - skeletal dysplasia } \\ & \text { - insulin resistance } \\ & \text { - diabetes } \\ & \text { - dyslipidaemia }\end{aligned}$

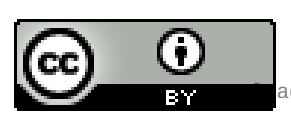

This work is licensed under a Creative Commons Attribution 3.0 Unported License. 


\section{Introduction}

Insulin action and linear growth are intimately linked, with crosstalk between insulin action and growth hormone/IGF1 signalling at multiple levels in liver and other tissues. This is attested to by the insulin resistance reported in the context of mutations in either the IGF1 receptor (Gannage-Yared et al. 2013) or the IGF acid labile subunit in humans (Hogler et al. 2014) and, conversely, by the marked linear growth impairment caused by biallelic INSR mutations (Semple et al. 2010, 2011). However growth and insulin action have links beyond these well-established endocrine mechanisms. Indeed, a subset of forms of primordial dwarfism caused by cellular defects with no obvious link to insulin action also feature severe dyslipidaemic insulin resistance. These include osteodysplastic primordial dwarfism of Majewski type 2 (MOPDII) (Huang-Doran et al. 2011), caused by recessive mutations in the pericentrosomal protein PCNT (Rauch et al. 2008), a recently described form of dwarfism caused by defects in the SUMO ligase NSMCE2 (Payne et al. 2014a), which is involved in accurate DNA repair in replicating cells, and Bloom syndrome (Diaz et al. 2006), caused by mutations in the BLM DNA helicase (Ellis et al. 1995).

Most known forms of primordial dwarfism, however, have not been reported to be associated with insulin resistance. Moreover, other pleiotropic disorders have been described affecting either another large component of the centrosome, ALMS1, or another DNA helicase, WRN. Interestingly both of the resulting clinical syndromes, namely Alström syndrome and Werner syndrome, also feature severe insulin resistance and dyslipidaemia (Marshall et al. 2011, Watanabe et al. 2013). Increasing understanding of the mechanistic links among insulin action, growth, and cell autonomous defects in centrosomal function and DNA damage repair is likely to yield novel insights into the pathogenesis of systemic insulin resistance, which a substantial body of evidence has implicated as an important mediator of the link between obesity and pathologies such as type 2 diabetes, fatty liver disease, polycystic ovary syndrome and some cancers (Savage \& Semple 2010, Parker et al. 2011, Semple et al. 2011, Stears et al. 2012).

A new form of primordial dwarfism caused by a homozygous genetic defect in the luminal centriolar protein POC1A was recently described (Sarig et al. 2012, Shaheen et al. 2012, Koparir et al. 2015), and we now add to this by reporting a further affected patient with a frameshift mutation predicted to affect two out of three POC1A isoforms. This was associated with supernumerary centrosomes and multipolar mitotic spindles in primary cells, and with sustained and extreme dyslipidaemic insulin resistance. This adds a further piece to the puzzle regarding the pathogenic mechanisms linking centrosome/ciliary dysfunction and metabolic disease.

\section{Materials and methods}

\section{Patients and clinical studies}

All human studies were approved by the United Kingdom National Health Service Research Ethics Committee. The proband and family provided written informed consent, and studies were conducted in accordance with the principles of the Declaration of Helsinki. For biochemical assays, venous blood was drawn in the fasting state and plasma immediately extracted then stored at $-20^{\circ} \mathrm{C}$. Insulin, leptin and adiponectin were assayed using twostep, time-resolved AutoDELFIA immunoassays described previously (Semple et al. 2006). Other analytes were determined in accredited clinical diagnostic laboratories of the referring hospital. For oral glucose tolerance testing, $1.75 \mathrm{~g}$ glucose $/ \mathrm{kg}$ was administered after a $10 \mathrm{~h}$ fast and blood samples taken at the times indicated for determination of plasma glucose and insulin.

Body composition was measured by Lunar Prodigy dual-energy X-ray absorptiometry (GE Lunar). Abdominal fat distribution, including hepatic triglyceride content, was assessed by proton magnetic resonance spectroscopy (MRS), using a Siemens 3T Tim Trio MR scanner (Siemens Healthcare, Erlangen, Germany). All protocols have been described in detail previously (Semple et al. 2009).

\section{Genetic studies}

Whole exome sequencing and variant calling of genomic DNA extracted from peripheral blood leukocytes was performed as part of the UK10K Project as described previously (Futema et al. 2012). Calls were annotated with 1000 Genomes Phase 1 integrated callset v3 allele frequencies (ftp://ftp.1000genomes.ebi.ac.uk/vol1/ftp/ phase1/analysis_results/integrated_call_sets) and the NCBI dbSNP database build 137 (ftp://ftp.ncbi.nih.gov/ snp/organisms/human_9606/database/b137_archive/ organism_data/). Functional annotation was added using Ensembl Variant Effect Predictor v2.8 (McLaren et al. 2010) against Ensembl build 70. Variants were further annotated with allele frequencies from the Exome Variant Server,

Published by Bioscientifica Ltd 
NHLBI GO Exome Sequencing Project (ESP), Seattle, WA (URL: http://evs.gs.washington.edu/EVS/) (April 2013), the UK10K Cohorts (REL-2012-06-02) and Exome (REL2013-04-20) groups (www.UK10K.org) and exome-wide sequencing data from 409 control individuals from the CoLaus Cohort (Firmann et al. 2008). Raw exome sequence data is available from the European Genome-Phenome Archive (https://www.ebi.ac.uk/ega/home; accession numbers EGAN00001083652 and EGAN00001028741).

Variants were defined as potentially functional if they were non-synonymous, resulted in loss or gain of a stop codon or a frameshift, or occurred within essential splice sites. Those unlikely to have a functional impact were removed, as were all heterozygous variants and those found with a non-reference allele frequency $>1 \%$ in individuals of European descent from any of the annotated control populations (1000 genomes, UK10K cohorts and exomes, NHLBI exomes and CoLaus exomes). Variants present in the unaffected sister or failing to pass the quality filters were also removed. The remaining variants were then evaluated as described previously (Payne et al. 2014b). Comments on predicted alternative protein products of the POC1A gene are based on Ensembl build 70 .

\section{Cell culture}

Dermal fibroblasts derived from skin biopsy of proband and healthy controls were grown in DMEM (D6546, Sigma) supplemented with 10\% FCS (HyClone), $4 \mathrm{mM}$ L-glutamine (G7513, Sigma), 1\% penicillin-streptomycin (P0781, Sigma) in a humidified incubator $\left(37^{\circ} \mathrm{C}, 5 \% \mathrm{CO}_{2}\right)$. Primary cilia formation was induced by culturing cells in serum-free medium for $24 \mathrm{~h}$.

\section{cDNA synthesis and sequencing}

Total RNA was prepared using RNeasy Mini Kits (Qiagen) with a DNase digestion step to eliminate contaminating DNA, and was quantified on a NanoDrop ND-1000 (Thermo Scientific, Waltham, MA, USA). First strand cDNA was reverse-transcribed from $400 \mathrm{ng}$ of total RNA using an ImProm-II Reverse Transcription System (Promega) with random hexamer as the primer, according to the manufacturer's protocol. PCR was carried out with a GoTaq Green Master Mix (Promega) and gene-specific primers. Primers were custom-designed and synthesized by Sigma: POC1A forward primer $\left(5^{\prime}-3^{\prime}\right)$ GAACAAGTGATGGTTTGGAAGA, POC1A reverse primer $\left(5^{\prime}-3^{\prime}\right)$ CCCAAGTCCCATGGTACAAA. PCR products were examined by $1 \%$ agarose gel followed by Exo1/SAP treatment. Sequencing reactions were performed with a POC1A forward primer (5'-3') CAGAAGTGGACTTCCCTGTC and the preceding reverse primer.

\section{Western blotting}

Cells were washed with ice-cold PBS and harvested in M-PER Mammalian Protein Extraction reagent (Thermo Scientific) containing protease inhibitor mini complete cocktail (Roche) at a 1:7 ratio. Proteins were mixed with an equal volume of Laemmli sample buffer (\#161-0737, BioRad), denatured at $95^{\circ} \mathrm{C}$ and resolved by SDS-PAGE before transfer to polyvinylidene-fluoride membranes using the iBlot system (Invitrogen). Blots were blocked with 5\% BSA in TBST (50 mM Tris-HCl, pH7.6, $150 \mathrm{mM} \mathrm{NaCl}, 0.1 \%$ Tween-20) and probed overnight at $4{ }^{\circ} \mathrm{C}$ with anti-POC1A (HPA040600, Sigma) or anti-calnexin (ab75801, Abcam, Cambridge, UK) antibody. Bound primary antibody was detected by HRP conjugated secondary antibodies, followed by Immobilon Western chemiluminescent HRP substrate (WBKLS0500, Millipore, Watford, UK).

\section{Immunofluorescence analysis}

Cells on coverslips were fixed in 4\% paraformaldehyde in PBS for 10 min followed by one wash with PBS, permeabilization in $0.2 \%$ Triton X-100 in PBS for $5 \mathrm{~min}$, and three washes with PBS (5 min each wash). Coverslips were blocked with blocking buffer (10\% horse serum, 1\% BSA, $\left.0.02 \% \mathrm{NaN}_{3}, 1 \times \mathrm{PBS}\right)$ for $1 \mathrm{~h}$, washed with PBS and incubated with anti-ALMS1 (ab84892, Abcam) and antiacetylated tubulin (T7451, Sigma-Aldrich) in 1\% BSA in PBS overnight at $4{ }^{\circ} \mathrm{C}$. After washing with PBS, the cells were incubated with 1:1000 dilution of Alexa Fluor 488 goat anti-mouse IgG (A11001, Invitrogen) and Alexa Fluor 555 goat anti-rabbit IgG (A21430, Invitrogen) for $45 \mathrm{~min}$ at room temperature in the dark, washed with PBS, mounted on glass slides using the ProLong Gold Antifade Reagent with DAPI (P36931, Invitrogen) and inspected with a Zeiss LSM510 Meta confocal laser scanning microscope. For detecting DNA damage, anti- $\gamma \mathrm{H} 2 \mathrm{AX}$ antibody (05-636, Millipore) was used according to the above method except that TBS (50 mM Tris-HCl (pH7.4), $150 \mathrm{mM} \mathrm{NaCl}$ ) instead of PBS was used.

\section{Statistical analysis}

Statistical analyses were performed in GraphPad Prism 5.0 (GraphPad Software, San Diego, CA, USA). Statistical

Published by Bioscientifica Lto 
significance was determined by pairwise comparisons using a two-tailed unpaired Student's $t$-test with a $P<0.05$ being considered significant. All data are presented as means \pm s.E.M.

\section{Results}

\section{Case history}

The proband is a 26 year-old woman from Italy born at full term to healthy parents who were first cousins. Her birth weight was $2.08 \mathrm{~kg}$ (-3.1 SDS). The placenta was small and calcified, and surgical repair of a patent ductus arteriosus was performed at 5 months. Linear growth was reported to be retarded throughout development. Menarche occurred precociously at 8.5 years old, and acanthosis nigricans was first noted by the proband's mother at around the same age. At 12.7 years, oral glucose tolerance testing revealed fasting hyperinsulinaemia, with plasma insulin rising to over $5000 \mathrm{pmol} / \mathrm{l}$ after a glucose challenge. Fasting glucose and glucose tolerance were normal (Table 1).

Radiological investigation at 11.5 years showed evidence of skeletal dysplasia, with widespread narrowing of the medullary canals of the long bones. The acetabulum appeared dysplastic with a short, thick femoral neck. Middle and distal phalanges were shortened, and bone age was 14.5 years. There was reduced lumbar lordosis, and vertebral bodies had a 'dog-like' appearance. There was modestly reduced interpeduncular distance at the base of the lumbar spine, and some lumbosacral transitional vertebrae were present. Chest and skull radiographs were normal.

On examination at 21 years, height was $1.39 \mathrm{~m}(-4.1$ SDS), with relative macrocephaly (head circumference +2 SDS). There were no neurodevelopmental abnormalities.
Facial dysmorphism included micrognathia, a prominent nose, posteriorly rotated low set ears, and brachydactyly with widespread flexural acanthosis nigricans extending to the nasolabial crease, perioral and periocular regions. There was moderate facial hirsutism. No nail hypoplasia nor alopecia was found (Fig. 1). BMI was $24.5 \mathrm{~kg} / \mathrm{m}^{2}$, with a mildly centripetal pattern of adiposity. Oral glucose tolerance testing revealed sustained fasting and postprandial severe hyperinsulinaemia, again with normal fasting glucose and impaired glucose tolerance (Table 1). There was severe hypertriglyceridaemia and a low HDL cholesterol, plasma leptin was elevated and adiponectin severely suppressed, and total testosterone was elevated despite a low serum SHBG. Despite these severe biochemical features of insulin resistance, most commonly seen in the face of morbid obesity, the total body fat content, assessed using dual energy X-ray absorptiometry, was normal. Nevertheless MRS revealed grossly elevated hepatic triglyceride content relative to water (Table 2). Metformin was commenced and titrated to $1.5 \mathrm{~g} /$ day in divided doses.

At 26 years, oral glucose tolerance testing confirmed persisting extreme insulin resistance (Table 1), still with normal glucose tolerance, while treated with $1.5 \mathrm{~g} /$ day of metformin. Severe dyslipidaemia also persisted, with serum triglycerides of $25.0 \mathrm{mmol} / \mathrm{l}$, and HDL cholesterol $0.57 \mathrm{mmol} / 1$. Mildly elevated serum ALT, AST and $\gamma \mathrm{GT}$ were consistent once again with significant fatty liver.

The proband's unaffected younger sister had a height of $1.63 \mathrm{~cm}$ at 18 years ( -0.09 SDS). Both parents were of normal stature. There was no evidence of short stature nor severe insulin resistance within the extended family. However, the proband's father and both grandmothers, of lean build, were reportedly diagnosed with type 2 diabetes mellitus in their sixth decade. The proband's father had a normal HbA1c on therapy with metformin

Table 1 Results of serial oral glucose tolerance testing of the proband

\begin{tabular}{|c|c|c|}
\hline \multirow[b]{2}{*}{ Time (min) } & \multicolumn{2}{|c|}{12.7 years (no treatment) } \\
\hline & Glucose $(\mathrm{mmol} / \mathrm{l})$ & Insulin (pmol/l) \\
\hline-30 & - & - \\
\hline 0 & 4.2 & 532 \\
\hline 10 & - & - \\
\hline 30 & 6.2 & 4375 \\
\hline 60 & 6.2 & 4494 \\
\hline 90 & 5.3 & 5005 \\
\hline 120 & 4.6 & 3514 \\
\hline 150 & - & - \\
\hline 180 & - & - \\
\hline Fasting reference range & $<5.6$ & $<60$ \\
\hline
\end{tabular}

\begin{tabular}{ccc}
\multicolumn{2}{c}{$\mathbf{2 1 . 3}$ years (no treatment) } \\
\cline { 1 - 1 } Glucose $(\mathrm{mmol} / \mathrm{l})$ & & Insulin (pmol/l) \\
\cline { 1 - 1 } 5.4 & & 201 \\
5.1 & 1675 \\
5.2 & 1661 \\
9.4 & 5596 \\
10.2 & 10290 \\
11.3 & 12060 \\
9.1 & 9985 \\
8.3 & 10300 \\
7.3 & 8089 \\
$<5.6$ & $<60$
\end{tabular}

26.4 years (metformin 1.5 g/day)

Glucose $(\mathrm{mmol} / \mathrm{l}) \quad$ Insulin $(\mathrm{pmol} / \mathrm{l})$

-

$-$

7.5

8.6

6.9

$-$

$<\overline{5} .6$

219

1416

1978

3095

5108

$-$

$<60$ 

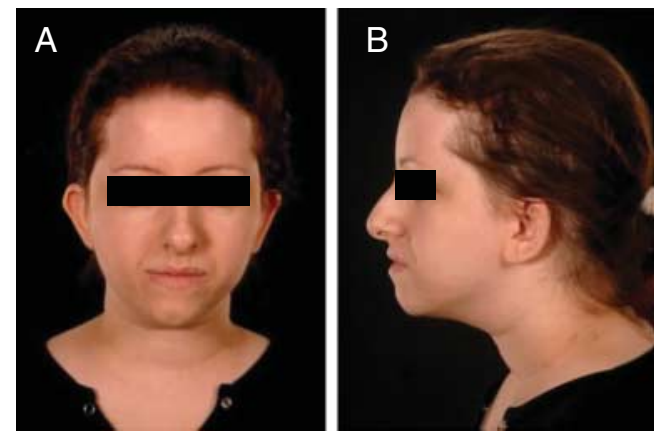

Figure 1

Clinical appearance of proband. (A and B) Facial appearance of the proband showing facial dysmorphism including a triangular face with a prominent nose, posteriorly rotated low set ears, acanthosis nigricans extending to the nasolabial crease, perioral and periocular regions, and facial hirsutism. No alopecia was found. Written informed consent was given by the proband for use of clinical images.

only, while oral glucose tolerance testing in the proband's mother and sister revealed normal levels of both glucose and insulin at baseline and after $120 \mathrm{~min}$.

\section{Genetic analysis}

Given parental consanguinity, it was hypothesised that the syndrome was caused by a homozygous lossof-function mutation not shared by the proband's unaffected sister. To address this, exome-wide sequencing of the proband and her sister was performed. The resulting list of homozygous variants was filtered to exclude those unlikely to alter protein function, also homozygous in the sister, or common in control populations. Seven such variants were thus identified and validated by Sanger sequencing (Supplementary Table 1, see section on supplementary data given at the end of this article), among which was a novel single nucleotide deletion (c.1048delC) in POC1A (NM_001161580) that was absent from all control populations and was predicted to cause a frameshift and premature truncation of the protein product (p.Q250Rfs*4) (Fig. 2A). Both parents and sister were heterozygous for this deletion.

POC1A is a centrosomal protein that localises to both the inner luminal walls and proximal ends of centrioles (Brito et al. 2012) and plays a role in centriole formation and maintenance (Venoux et al. 2013). It harbours seven $\mathrm{N}$ terminal WD40 domains in series, commonly involved in the assembly of multi-protein complexes, followed by a spacer region and $\mathrm{C}$ terminal coiled coil domain that is removed by the frameshift mutation we identified (Fig. 2A).
Although we hypothesised a recessive disorder given parental consanguinity, as an additional screen for alternative causes of insulin resistance we also examined variants in other genes previously shown to produce severe insulin resistance (INSR, PIK3R1, AKT2, TBC1D4, LMNA, PPARG, BSCL2, AGPAT2, PTRF, CAV1, PCNT, NSMCE2, ALMS1, WRN, and BLM). After removing any variants found in the listed genes with a non-reference allele frequency $>1 \%$ in any of the control populations, or unlikely to have a functional impact (as determined previously), only two variants remained (ALMS1 p.Ile3144Val and AGPAT2 p.Arg159Cys). Both of these heterozygous variants were also seen in at least two of the control populations (albeit with a non-reference allele frequency below 1\%) and are extremely unlikely to be causal on their own given the very well-established recessive mode of inheritance for both the associated disorders. As three recent studies associated different homozygous mutations in POC1A with primordial dwarfism and facial dysmorphism highly similar to that of the proband in this case (Sarig et al. 2012, Shaheen et al. 2012, Koparir et al. 2015), this rare frameshift variant was deemed to be the cause of the syndrome we describe.

\section{Primary cell studies}

Primary dermal fibroblast cultures were established from the proband and grew well. No deficit in

Table 2 Growth and metabolic parameters of the proband at 21.3 years old. Ranges for leptin and adiponectin are BMI and sex-specific

\section{Parameter}

Height (m) (SDS)

Weight $(\mathrm{kg})(\mathrm{SDS})$

BMI $\left(\mathrm{kg} / \mathrm{m}^{2}\right)$ (SDS)

Fat (\%) (SDS)

Waist:hip

$\mathrm{HbA1c}(\mathrm{mmol} / \mathrm{mol})$

Leptin (mg/l)

Adiponectin (mg/l)

SHBG (mmol/l)

Testosterone $(\mathrm{nmol} / \mathrm{l})$

Triglycerides $(\mathrm{mmol} / \mathrm{l})$

HDL-cholesterol (mmol/l)

ALT (U/I)

$\gamma \mathrm{GT}(\mathrm{U} / \mathrm{I})$

Liver fat $(\%)^{\mathrm{b}}$

\begin{tabular}{c}
\hline Value \\
\hline $1.39(-4.1)$ \\
$46.8(+1.6)$ \\
$24.5(+0.8)$ \\
$34.3(+0.8)$ \\
0.84 \\
31 \\
32.1 \\
0.9 \\
19.7 \\
3.8 \\
5.0 \\
0.69 \\
102 \\
51 \\
108
\end{tabular}

Reference range ${ }^{a}$

\section{NA}

NA

NA

NA

$<0.85$

20-38

2.4-24.4

2.6-12.6

26-110

$<2.2$

$<1.7$

$>1.1$

$0-50$

$0-31$

$<8$

SHBG, sex hormone-binding globulin; ALT, alanine aminotransferase; $\gamma \mathrm{GT}, \gamma$-glutamyl transpeptidase.

${ }^{\mathrm{a}}$ Fasting where appropriate.

${ }^{\mathrm{b}}$ The size of the magnetic resonance spectroscopy signal for triglyceride protons expressed as $\%$ of the water proto signal. 
A

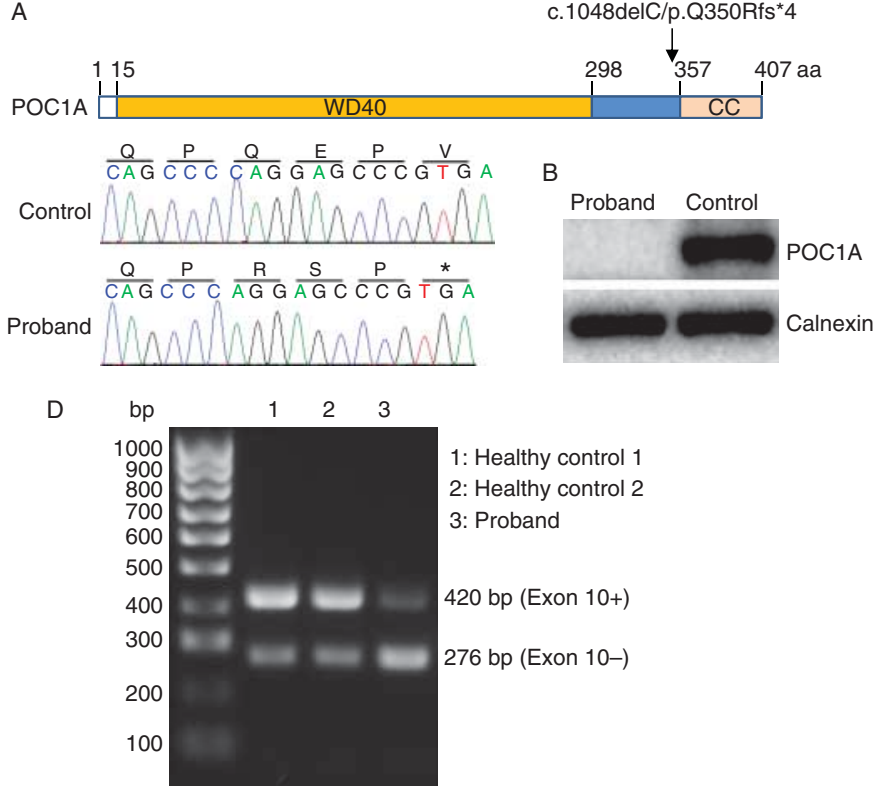

C

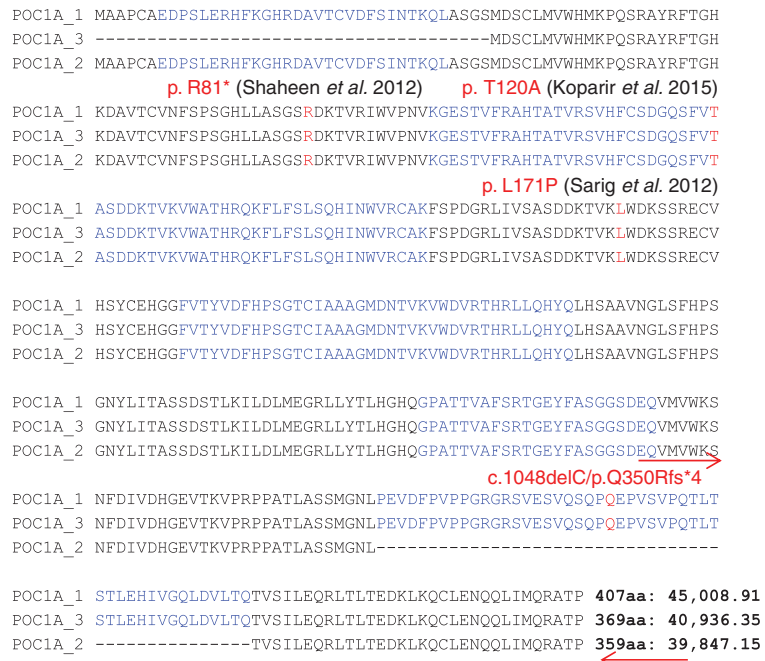

\section{Figure 2}

POC1A frameshift mutation and detection of its protein and mRNA expression. (A) Schematic of POC1A showing that the protein contains a WD40-repeat domain and a coiled coil (CC) domain separated by a spacer region. The mutation site is indicated by the arrow and the deletion mutation and resulting frameshift are shown in a sequence chromatogram. (B) Western blot analysis of primary dermal fibroblast lysates of the proband and of a healthy control. (C) Three splicing variants of POC1A were aligned and mutation in the proband in this study as well as

insulin-stimulated AKT phosphorylation was observed, consistent with a lack of any defect in proximal insulin signalling (Supplementary Figure 1, see section on supplementary data given at the end of this article). However, western blotting failed to detect expression of either full length WT or truncated POC1A (Fig. 2B). Two shorter isoforms of POC1A have been predicted, one lacking the N-terminal 38 amino acids due to alternative start codon usage, and the other lacking 48 amino acids due to alternative splicing of exon 10. Exon 10 encodes a large part of the spacer region and a small part of the coiled coil domain of the longest isoform of POC1A (407 amino acids long) (Fig. 2C), and is the site of the mutation identified in this study. PCR amplification of cDNA using primers flanking exon 10 produced two products of the sizes expected for exon $10+$ and exon $10-$ transcripts (Fig. 2D), suggesting that a WT exon 10 - isoform may still be produced. Indeed, in two healthy control cell lines the predominant PCR product was from the exon $10+$ isoform whereas in the patient cells the predominant PCR product was from the exon $10-$ isoform (Fig. 2D). The antibody used in immunoblotting studies was raised mutations described in three previous reports were indicated in red letters. Amino acids encoded by 11 exons were indicated in black and blue letters. Numbers of amino acids and the predicted molecular sizes of each isoforms were indicated at the end of each isoform sequence. Locations of primers used for PCR to reveal the expected alternative splicing of exon 10 were indicated by red arrows (the reverse primer was in the $3^{\prime}$ UTR). (D) PCR products using primers as indicated in (C) were revealed by an agarose gel $(1 \%)$ with SYBR Safe in the gel to stain amplified DNAs.

against a region of which a third is encoded by exon 10, and so it is plausible that this putative shorter protein is indeed expressed, but not detected in the proband's cells.

As mutations in POC1A have been associated with centrosome amplification (Sarig et al. 2012, Shaheen et al. 2012, Koparir et al. 2015), we next examined centrosome number in the proband's fibroblasts using immunostaining with antibody against ALMS1, a protein localized to the proximal ends of centrioles (Knorz et al. 2010). About $12 \%$ of interphase cells showed centrosomal amplification with centrosome number ranging from 3 to 10 per cell. During the mitotic phase, around 39\% of cells showed multipolar spindle formation (Fig. 3A and Supplementary Figure 2, see section on supplementary data given at the end of this article). In both cases, proband cells showed over tenfold increase in the frequency of centrosomal amplification and multipolar spindle formation compared to the control cells.

Despite evidence of centrosome amplification, the proliferation rate of the proband's cells, measured by BrdU incorporation, did not show any difference compared to the control cells (Supplementary Figure 3,

Published by Bioscientifica Ltd 

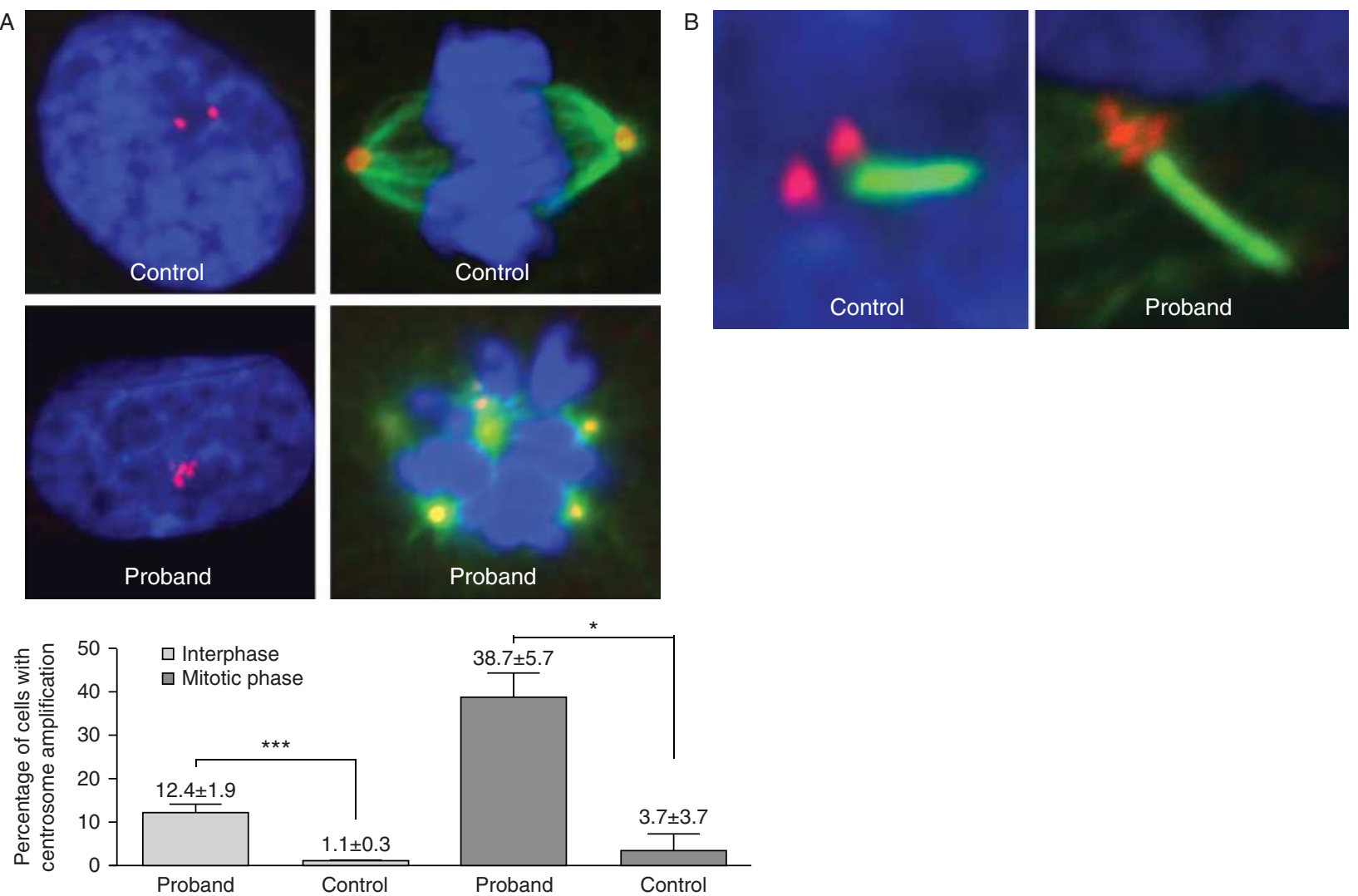

\section{Figure 3}

POC1A frameshift mutation and associated centrosome and basal body amplification. (A) Immunofluorescence analysis of dermal fibroblasts shows normal centrosome duplication and bipolar spindle formation in healthy control cells and centrosome amplification and multipolar spindle formation in proband cells. Centrosomes (red) were revealed using anti-ALMS1 antibodies and spindle fibres (green) were revealed using anti-acetylated tubulin antibodies together with appropriate Alexa Fluor conjugated secondary antibodies. Nuclei (blue) were revealed with DAPI.

see section on supplementary data given at the end of this article). Although centrosome amplification can lead to multipolar spindle formation, we found that primary cilia in proband dermal fibroblasts were of normal length and frequency (Supplementary Figure 4), with multiple centrosomes always clustered at the basal body of a single cilium (Fig. 3B).

Supernumerary centrioles/centrosomes may arise due to overduplication, aberrant de novo synthesis, mitotic skipping, cleavage failure or cell fusion (Anderhub et al. 2012). DNA content analysis by FACS revealed a cell cycle distribution profile for the proband's dermal fibroblasts that was indistinguishable from healthy control cells (Supplementary Figure 5A), suggesting that centrosome amplification in the proband's cells was due to overduplication rather than cleavage failure, mitotic skipping
Bar charts show percentages of cells with centrosome amplification in interphase and mitotic phase. Error bars represent the S.E.M. Asterisks indicate statistical significance $(* P<0.05, * * * P<0.001)$ as analysed by the Student's t-test. (B) Example of amplification of the basal body (red, stained with anti-ALMS1 antibodies) of a primary cilium (green, stained with anti-acetylated tubulin) in the proband's dermal fibroblasts compared to the normal appearance of a healthy control.

or cell fusion, as these would produce cells with 8N DNA content. In line with this, karyotype analysis in the proband fibroblasts was normal (Supplementary Figure 5B).

As for primary cilia, where centrosome clustering was seen in the region of the basal body (Fig. 3B), clusters of centrosomes were also seen at many mitotic spindles (Fig. 4A). Centrosome clustering prevents multipolar cell division, however, it can lead to syntelic or merotelic kinetochore attachments which can lead in turn to chromosome mis-segregation (Anderhub et al. 2012, Vitre \& Cleveland 2012). One consequence of chromosome mis-segregation, if not corrected by the activity of Aurora-B, is the formation of anaphase bridges or lagging chromosomes, detectable as micronuclei (Anderhub et al. 2012, Vitre \& Cleveland 2012). These abnormalities were

Published by Bioscientifica Ltd 

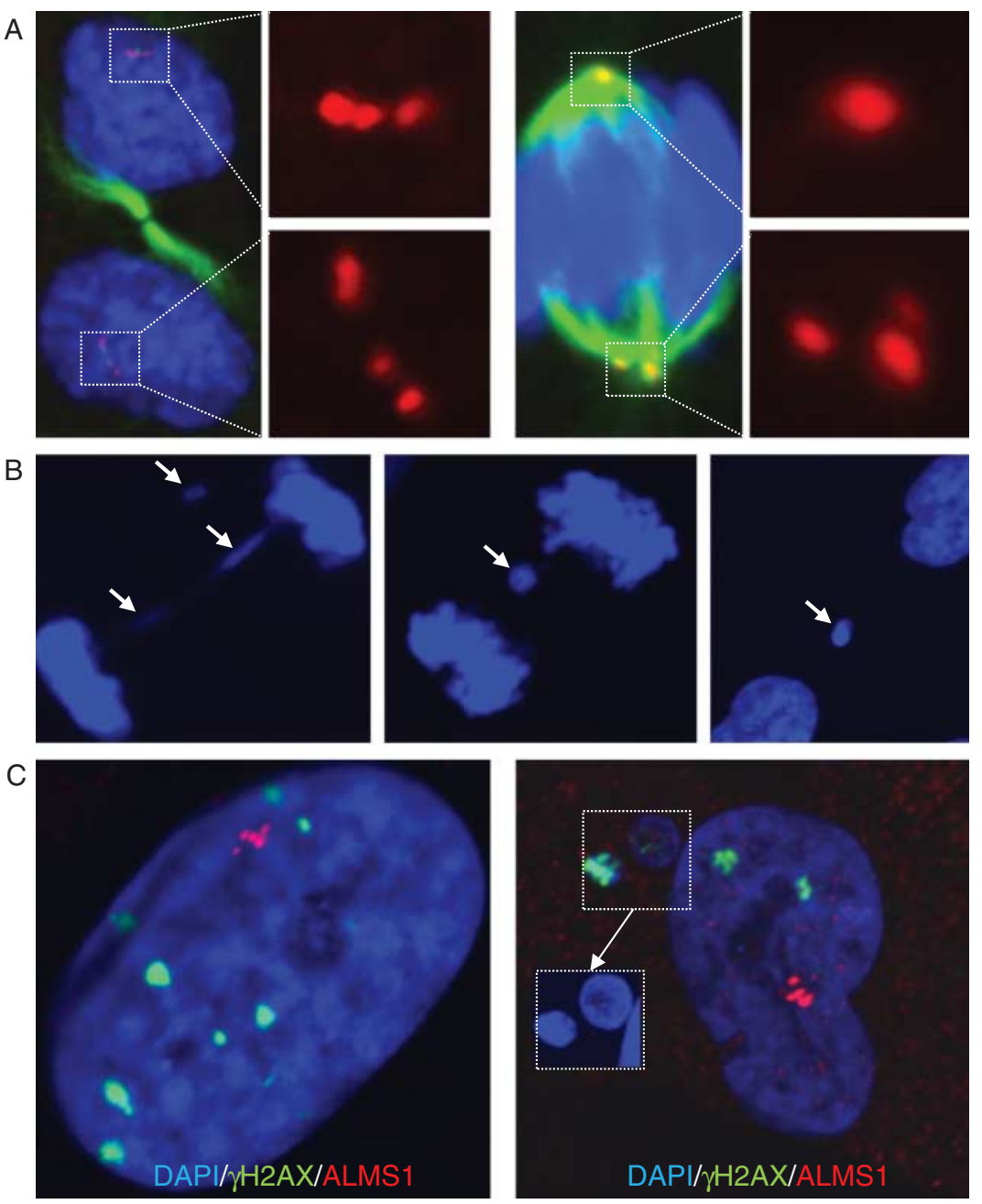

\section{Figure 4}

Centrosome clustering and evidence of DNA damage in cells harbouring centrosome amplification. (A) Centrosome clustering in proband dermal fibroblasts, with either even (left) or uneven partitioning between spindle poles (right). Immunostaining was performed using antibodies as in Fig. 3.

observed at a higher level in the proband cells with centrosome amplification (Fig. 4B) with micronuclei staining avidly for anti- $\gamma \mathrm{H} 2 \mathrm{AX}$, suggesting the presence of double stranded DNA breaks (Fig. 4C). This was consistent with Western blotting which showed $\gamma \mathrm{H} 2 \mathrm{AX}$ to be higher in proband cells than in those from two healthy control individuals (Supplementary Figure 6, see section on supplementary data given at the end of this article).

\section{Discussion}

We used exome-wide sequencing to investigate a single proband with consanguineous parents and a rare
(B) Example of an anaphase bridge and resulting micronuclei formation in proband cells. (C) DNA damage foci in the nuclei and micronuclei of proband dermal fibroblasts with centrosome amplification revealed by immunofluorescence staining using anti- $\gamma \mathrm{H} 2 \mathrm{AX}$ antibody.

syndrome of primordial dwarfism, facial dysmorphism, skeletal dysplasia, and extreme, dyslipidaemic insulin resistance. We identified seven rare, functional homozygous variants, among which was a frameshift mutation in POC1A, which was associated during the course of our study by three groups with a similar syndrome in five consanguineous families from the Middle East (Sarig et al. 2012, Shaheen et al. 2012, Koparir et al. 2015). Three of these families harboured one of two missense mutations (Sarig et al. 2012, Koparir et al. 2015), while the other three families had in common an early nonsense mutation, although cellular studies suggested significant translational readthrough of the mutation (Shaheen et al. 2012).

Published by Bioscientifica Ltd 
On the basis of the close similarity among the reported phenotypes, it is highly likely that the new POC1A variant is the cause of the syndrome we describe. The growth parameters, facial dysmorphism and skeletal features of the proband in this report are highly similar to other published cases (Koparir et al. 2015), and although the large majority of patients reported to date are of prepubertal age, one other patient has been described with precocious puberty (Koparir et al. 2015), which may play a role in the short final height of the proband we describe. There are significant phenotypic differences among patients reported to date. Most prominently, the proband we describe had no ectodermal dysplasia, reported in three of four families to date (Shaheen et al. 2012, Koparir et al. 2015), and, conversely, did have extreme, dyslipidaemic insulin resistance, which was not clearly described in other reports. Differing genetic backgrounds may contribute to these differences, as suggested by the occurrence of type 2 diabetes of onset in middle age in several members of the family we describe, albeit without the extreme insulin resistance of the proband. We could not attribute this to rare variants in other genes previously associated with Mendelian insulin resistance, and nor were there any obvious functional interactions between POC1A and other genes harbouring rare homozygous variants in this study. Nevertheless such interactions cannot be formally excluded in an individual patient, and nor can an effect of heterozygous POC1A loss of function on susceptibility to type 2 diabetes be ruled out based on the data we present.

Some of the phenotypic differences may alternatively be explained by the differing nature of the POC $1 \mathrm{~A}$ mutations involved in each report: previous cases were attributed to POC1A hypomorphism due to partial readthrough of a premature nonsense mutation in one report (Shaheen et al. 2012), and to rare missense mutations in two others (Sarig et al. 2012, Koparir et al. 2015). In all cases, all protein products of the gene were predicted to be affected. In the current report, in contrast, we identified a frameshift mutation predicted to abolish expression of two out of three protein isofoms. We provide evidence that one of the predicted POC1A mRNA species, lacking exon 10 and thus the frameshift mutation, is indeed expressed, although formal proof of the existence of the corresponding exon $10-$ isoform of POC1A protein is lacking. The exon 10 - isoform retains the WD40 domain, required for targeting POC1A to centrioles but lacks almost half of the coiled coil POC1 domain, implicated in interactions with other proteins (Keller et al. 2009). It is therefore conceivable that WT exon 10- POC1A protein, if expressed, can partially compensate for the loss of the exon $10+$ isoforms in our patient. Examination of further cases will be required to build a more robust view of genotype-phenotype correlation.

Despite the lack of detailed metabolic assessment reported for other cases, two affected males in one report were said to have developed unusually early type 2 diabetes (Shalev et al. 2012), at 20 and 24 years old, a common feature of severe insulin resistance. Moreover, a further patient has been identified with clearly demonstrated severely dyslipidaemic insulin resistance (Prof Fowzan Alkuraya, King Faisal Specialist Hospital and Research Center, Riyadh, personal communication), strongly suggesting that severe insulin resistance is a significant component of the syndrome associated with genetic defects in POC1A. It is notable that the majority of patients described to date are of prepubertal age (Koparir et al. 2015), when even many highly penetrant forms of Mendelian severe insulin resistance are yet to be clinically expressed, and we suggest that metabolic evaluation for severe insulin resistance is warranted for patients with this syndrome from puberty onwards.

The insulin resistance subphenotype we report, including severe dyslipidaemia, suppressed adiponectin and severe fatty liver is closely similar to that shared by lipodystrophy (Semple et al. 2011) and severe obesity, and also, interestingly, by both Alström syndrome (Collin et al. 2002, Hearn et al. 2002) and MOPDII (Rauch et al. 2008, Willems et al. 2010), each accounted for by defects in large centriolar or pericentriolar proteins. Alström syndrome is widely regarded as a ciliopathy (Girard \& Petrovsky 2011), while in MOPDII the core cellular defect appears to be inefficient mitosis (Klingseisen \& Jackson 2011, Rauch 2011). A wide number of other primary ciliopathies, notably the Bardet Biedl syndrome group, are known, but it is not clear that they feature insulin resistance that is out of proportion to the degree of obesity, while most forms of primordial dwarfism have also not been associated with severe insulin resistance. It thus appears that only a subset of genetic defects affecting the centrosome give rise to extreme, dyslipidaemic insulin resistance. Further investigation of the pathogenesis of these unusual metabolic phenocopies of the prevalent metabolic syndrome is likely to yield novel information relevant to common disease as well as to these rare Mendelian disorders.

The primary cell phenotype we describe is in key respects consistent with previous studies, including centrosomal amplification and multipolar spindle formation, which we suggest is accounted for by primary

Published by Bioscientifica Lto 
centrosome overduplication. The threefold greater level of cells with supernumerary centrosomes seen during mitotis rather than during interphase is likely to be a consequence of prolonged mitosis due to kinetochore mis-attachment and chromosome mis-segregation (Silkworth et al. 2009). Although the mechanisms by which cells keep centrosome numbers constant during cell division remain to be fully elucidated it is generally believed that two distinct 'rules' apply. The first stipulates that centrosomes duplicate once only per cell cycle and the second that only one daughter centriole is produced per parent centriole (Nigg 2007). Although many centrosomal proteins have been identified, major centriole duplication factors are limited, with SAS6 and Plk4 being the best characterised (Kleylein-Sohn et al. 2007, Strnad et al. 2007, Holland et al. 2010). Overexpression of SAS6 and Plk4 causes near-simultaneous formation of multiple daughter centrioles (Kleylein-Sohn et al. 2007, Strnad et al. 2007), and Plk4 kinase activity normally limits centrosome overduplication by autoregulating its own stability (Holland et al. 2010, 2012). Investigating whether POC1A interacts functionally with these factors is an obvious route for further study.

Although we confirm multipolar spindle formation, multi-cilia formation was not observed. This is consistent with previous knockdown studies which showed that the paralogue $P O C 1 B$, but not $P O C 1 A$, is required for ciliogenesis in human cells (Pearson et al. 2009). Similarly, Venoux et al. (2013) recently observed that although $P O C 1 A$ and $P O C 1 B$ act together in human cells to ensure centriole integrity, only $P O C 1 B$ had additional, nonredundant functions in cell. On the other hand, ciliary formation was reported to be severely suppressed in primary skin cells from one previous patient studied, although sonic hedgehog (SHH) signalling in POC1Aknockdown cells was unaffected (Shaheen et al. 2012). Whether this discrepancy has a methodological basis, or whether it relates to the different molecular defects present in POC1A in these different studies, remains unclear.

Multiple centrosomes were always clustered at the basal body of a single cilium in our patient cells. Such centrosome clustering is believed to be a survival mechanism, permitting cells to avoid multipolar division (Ganem et al. 2009, Vitre \& Cleveland 2012) by undergoing pseudo-bipolar mitosis (Anderhub et al. 2012, Vitre $\&$ Cleveland 2012). Chromosome mis-segregation can also lead to aneuploidy and although karyotypic analysis failed to detect this in the proband cells, it remains possible that low levels of aneuploidy in vivo resulting from chromosome mis-segregation could play some role in causing cellular malfunction in our patient, for example via proteotoxic and energetic stress which can cause cell cycle arrest or apoptosis (Pfau \& Amon 2012).

In summary, we describe a novel frameshift mutation in POC1A in association with short stature, skeletal dysplasia and extreme, dyslipidaemic insulin resistance. The truncating mutation abolished expression of the mutation-bearing exon $10+$ isoform in patient, although the WT exon 10- isoform may still be expressed and thereby partially compensate for abolished expression of exon $10+$ isoforms. Primary dermal fibroblasts exhibited centrosome amplification with evidence of increased DNA damage, but no readily detectable aneuploidy.

Supplementary data

This is linked to the online version of the paper at http://dx.doi.org/10.1530/ JME-15-0090.

Declaration of interest

The authors declare that there is no conflict of interest that could be perceived as prejudicing the impartiality of the research reported.

\section{Funding}

This work was supported by the Wellcome Trust (grant numbers WT098498, WT098051, WT095515, and WT091310); the Medical Research Council (MRC_MC_UU_12012/5); the United Kingdom National Institute for Health Research (NIHR) Cambridge Biomedical Research Centre; and the EU/EFPIA Innovative Medicines Initiative Joint Undertaking (EMIF grant no 115372).

\section{Acknowledgements}

We are grateful for access to exome sequence data from the CoLaus cohort, which was sequenced as part of a partnership between the Wellcome Trust Sanger Institute, the CoLaus principal investigators and the Quantitative Sciences department of GlaxoSmithKline. We also thank the NHLBI GO Exome Sequencing Project and its ongoing studies which provided exome variant calls for comparison, namely the Lung GO (HL-102923), the WHI (HL-102924), the Broad GO (HL-102925), the Seattle GO (HL-102926) and the Heart GO (HL-103010) Sequencing Projects.

UK10K Consortium Management Committee: Principal Applicant, Richard Durbin, WTSI. Project Manager, Dawn Muddyman, WTSI. Cohorts Group: Nicole Soranzo, Cohorts Co-chair, WTSI; Nic Timpson, Cohorts Co-chair, ALSPAC - Bristol University; Tim Spector, TwinsUK, Kings College London; Brent Richards, McGill University. Neuro Group: Aarno Palotie, Neuro Group Co-chair, WTSI; Michael Owen, Neuro Group Co-chair, Cardiff University; Jeff Barrett, WTSI; Daniel Geschwind, UCLA. Rare Diseases Group: Matt Hurles, Rare Group Co-Chair, WTSI; David Fitzpatrick, Rare Group Co-Chair, MRC Edinburgh. Obesity Group: Inês Barroso, Obesity Group Co-chair, WTSI; Sadaf Farooqi, Obesity Group Co-chair, Cambridge University. Statistics Group: Eleftheria Zeggini, Statistics Group Co-chair, WTSI; Jeff Barrett, Statistics Group Co-chair, WTSI. Ethics Group: Jane Kaye, Ethics Group Co-chair, Oxford University; Karen Kennedy, WTSI. Production Group: Shane McCarthy, Production Co-chair, WTSI; Jim Stalker, Production Co-chair, WTSI; Cordelia Langford, Production Co-chair, WTSI; Thomas Keane, WTSI. A full list of Investigators and Working groups is available at http://www.uk10k.org/.

Published by Bioscientifica Ltd 


\section{References}

Anderhub SJ, Kramer A \& Maier B 2012 Centrosome amplification in tumorigenesis. Cancer Letters 322 8-17. (doi:10.1016/j.canlet.2012. 02.006)

Brito DA, Gouveia SM \& Bettencourt-Dias M 2012 Deconstructing the centriole: structure and number control. Current Opinion in Cell Biology 24 4-13. (doi:10.1016/j.ceb.2012.01.003)

Collin GB, Marshall JD, Ikeda A, So WV, Russell-Eggitt I, Maffei P, Beck S, Boerkoel CF, Sicolo N, Martin M et al. 2002 Mutations in ALMS1 cause obesity, type 2 diabetes and neurosensory degeneration in Alstrom syndrome. Nature Genetics 31 74-78. (doi:10.1038/ng867)

Diaz A, Vogiatzi MG, Sanz MM \& German J 2006 Evaluation of short stature, carbohydrate metabolism and other endocrinopathies in Bloom's syndrome. Hormone Research 66 111-117. (doi:10.1159/ 000093826)

Ellis NA, Groden J, Ye TZ, Straughen J, Lennon DJ, Ciocci S, Proytcheva M \& German J 1995 The Bloom's syndrome gene product is homologous to RecQ helicases. Cell 83 655-666. (doi:10.1016/0092-8674(95)90105-1)

Firmann M, Mayor V, Vidal PM, Bochud M, Pecoud A, Hayoz D, Paccaud F, Preisig M, Song KS, Yuan X et al. 2008 The CoLaus study: a populationbased study to investigate the epidemiology and genetic determinants of cardiovascular risk factors and metabolic syndrome. $B M C$ Cardiovascular Disorders 8 6. (doi:10.1186/1471-2261-8-6)

Futema M, Plagnol V, Whittall RA, Neil HA \& Humphries SE 2012 Use of targeted exome sequencing as a diagnostic tool for Familial Hypercholesterolaemia. Journal of Medical Genetics 49 644-649. (doi:10.1136/ jmedgenet-2012-101189)

Ganem NJ, Godinho SA \& Pellman D 2009 A mechanism linking extra centrosomes to chromosomal instability. Nature 460 278-282. (doi:10.1038/nature08136)

Gannage-Yared MH, Klammt J, Chouery E, Corbani S, Megarbane H, Abou Ghoch J, Choucair N, Pfaffle R \& Megarbane A 2013 Homozygous mutation of the IGF1 receptor gene in a patient with severe pre- and postnatal growth failure and congenital malformations. European Journal of Endocrinology/European Federation of Endocrine Societies 168 K1-K7. (doi:10.1530/EJE-12-0701)

Girard D \& Petrovsky N 2011 Alstrom syndrome: insights into the pathogenesis of metabolic disorders. Nature Reviews. Endocrinology 7 77-88. (doi:10.1038/nrendo.2010.210)

Hearn T, Renforth GL, Spalluto C, Hanley NA, Piper K, Brickwood S, White C, Connolly V, Taylor JF, Russell-Eggitt I et al. 2002 Mutation of ALMS1, a large gene with a tandem repeat encoding 47 amino acids, causes Alstrom syndrome. Nature Genetics 31 79-83. (doi:10.1038/ ag874)

Hogler W, Martin DD, Crabtree N, Nightingale P, Tomlinson J, Metherell L, Rosenfeld R, Hwa V, Rose S, Walker J et al. 2014 IGFALS gene dosage effects on serum IGF-I and glucose metabolism, body composition, bone growth in length and width, and the pharmacokinetics of recombinant human IGF-I administration. Journal of Clinical Endocrinology and Metabolism 99 E703-E712. (doi:10.1210/jc.2013-3718)

Holland AJ, Lan W, Niessen S, Hoover H \& Cleveland DW 2010 Polo-like kinase 4 kinase activity limits centrosome overduplication by autoregulating its own stability. Journal of Cell Biology 188 191-198. (doi:10.1083/jcb.200911102)

Holland AJ, Fachinetti D, Zhu Q, Bauer M, Verma IM, Nigg EA \& Cleveland DW 2012 The autoregulated instability of Polo-like kinase 4 limits centrosome duplication to once per cell cycle. Genes and Development 26 2684-2689. (doi:10.1101/gad.207027.112)

Huang-Doran I, Bicknell LS, Finucane FM, Rocha N, Porter KM, Tung YC, Szekeres F, Krook A, Nolan JJ, O'Driscoll M et al. 2011 Genetic defects in human pericentrin are associated with severe insulin resistance and diabetes. Diabetes 60 925-935. (doi:10.2337/db10-1334)

Keller LC, Geimer S, Romijn E, Yates J III, Zamora I \& Marshall WF 2009 Molecular architecture of the centriole proteome: the conserved WD40 domain protein POC1 is required for centriole duplication and length control. Molecular Biology of the Cell 20 1150-1166. (doi:10.1091/mbc. E08-06-0619)

Kleylein-Sohn J, Westendorf J, Le Clech M, Habedanck R, Stierhof YD \& Nigg EA 2007 Plk4-induced centriole biogenesis in human cells. Developmental Cell 13 190-202. (doi:10.1016/j.devcel.2007.07.002)

Klingseisen A \& Jackson AP 2011 Mechanisms and pathways of growth failure in primordial dwarfism. Genes and Development 25 2011-2024. (doi:10.1101/gad.169037)

Knorz VJ, Spalluto C, Lessard M, Purvis TL, Adigun FF, Collin GB, Hanley NA, Wilson DI \& Hearn T 2010 Centriolar association of ALMS1 and likely centrosomal functions of the ALMS motif-containing proteins C10orf90 and KIAA1731. Molecular Biology of the Cell 21 3617-3629. (doi:10.1091/mbc.E10-03-0246)

Koparir A, Karatas OF, Yuceturk B, Yuksel B, Bayrak AO, Gerdan OF, Sagiroglu MS, Gezdirici A, Kirimtay K, Selcuk E et al. 2015 Novel POC1A mutation in primordial dwarfism reveals new insights for centriole biogenesis. Human Molecular Genetics. (doi:10.1093/hmg/ddv261)

Marshall JD, Maffei P, Collin GB \& Naggert JK 2011 Alstrom syndrome: genetics and clinical overview. Current Genomics 12 225-235. (doi:10.2174/138920211795677912)

McLaren W, Pritchard B, Rios D, Chen Y, Flicek P \& Cunningham F 2010 Deriving the consequences of genomic variants with the Ensembl API and SNP Effect Predictor. Bioinformatics 26 2069-2070. (doi:10.1093/ bioinformatics/btq330)

Nigg EA 2007 Centrosome duplication: of rules and licenses. Trends in Cell Biology 17 215-221. (doi:10.1016/j.tcb.2007.03.003)

Parker VE, Savage DB, O'Rahilly S \& Semple RK 2011 Mechanistic insights into insulin resistance in the genetic era. Diabetic Medicine $\mathbf{2 8}$ 1476-1486. (doi:10.1111/j.1464-5491.2011.03463.x)

Payne F, Colnaghi R, Rocha N, Seth A, Harris J, Carpenter G, Bottomley WE Wheeler E, Wong S, Saudek V et al. 2014a Hypomorphism in human NSMCE2 linked to primordial dwarfism and insulin resistance. Journal of Clinical Investigation 124 4028-4038. (doi:10.1172/JCI73264)

Payne F, Lim K, Girousse A, Brown RJ, Kory N, Robbins A, Xue Y, Sleigh A, Cochran E, Adams C et al. 2014b Mutations disrupting the Kennedy phosphatidylcholine pathway in humans with congenital lipodystrophy and fatty liver disease. PNAS 111 8901-8906. (doi:10.1073/pnas. 1408523111)

Pearson CG, Osborn DP, Giddings TH Jr, Beales PL \& Winey M 2009 Basal body stability and ciliogenesis requires the conserved component Poc1. Journal of Cell Biology 187 905-920. (doi:10.1083/jcb.200908019)

Pfau SJ \& Amon A 2012 Chromosomal instability and aneuploidy in cancer: from yeast to man. ЕMBO Reports 13 515-527. (doi:10.1038/embor. 2012.65)

Rauch A 2011 The shortest of the short: pericentrin mutations and beyond. Best Practice \& Research. Clinical Endocrinology \& Metabolism 25 125-130. (doi:10.1016/j.beem.2010.10.015)

Rauch A, Thiel CT, Schindler D, Wick U, Crow YJ, Ekici AB, van Essen AJ, Goecke TO, Al-Gazali L, Chrzanowska KH et al. 2008 Mutations in the pericentrin (PCNT) gene cause primordial dwarfism. Science 319 816-819. (doi:10.1126/science.1151174)

Sarig O, Nahum S, Rapaport D, Ishida-Yamamoto A, Fuchs-Telem D, Qiaoli L, Cohen-Katsenelson K, Spiegel R, Nousbeck J, Israeli S et al. 2012 Short stature, onychodysplasia, facial dysmorphism, and hypotrichosis syndrome is caused by a POC1A mutation. American Journal of Human Genetics 91 337-342. (doi:10.1016/j.ajhg.2012.06.003)

Savage DB \& Semple RK 2010 Recent insights into fatty liver, metabolic dyslipidaemia and their links to insulin resistance. Current Opinion in Lipidology 21 329-336. (doi:10.1097/MOL.0b013e32833b7782)

Semple RK, Soos MA, Luan J, Mitchell CS, Wilson JC, Gurnell M, Cochran EK, Gorden P, Chatterjee VK, Wareham NJ et al. 2006 Elevated plasma adiponectin in humans with genetically defective insulin receptors. Journal of Clinical Endocrinology and Metabolism 91 3219-3223. (doi:10.1210/jc.2006-0166) 
Semple RK, Sleigh A, Murgatroyd PR, Adams CA, Bluck L, Jackson S, Vottero A, Kanabar D, Charlton-Menys V, Durrington P et al. 2009 Postreceptor insulin resistance contributes to human dyslipidemia and hepatic steatosis. Journal of Clinical Investigation 119 315-322. (doi:10.1172/CI37432)

Semple RK, Savage DB, Halsall DJ \& O'Rahilly S 2010 Syndromes of severe insulin resistance and/or lipodystrophy. In Genetic Diagnosis of Endocrine Disorders, 1st edn, pp 39-52. Eds RE Weiss \& S Refetoff. London: Elsevier.

Semple RK, Savage DB, Cochran EK, Gorden P \& O'Rahilly S 2011 Genetic syndromes of severe insulin resistance. Endocrine Reviews 32 498-514. (doi:10.1210/er.2010-0020)

Shaheen R, Faqeih E, Shamseldin HE, Noche RR, Sunker A, Alshammari MJ, Al-Sheddi T, Adly N, Al-Dosari MS, Megason SG et al. 2012 POC1A truncation mutation causes a ciliopathy in humans characterized by primordial dwarfism. American Journal of Human Genetics 91 330-336. (doi:10.1016/j.ajhg.2012.05.025)

Shalev SA, Spiegel R \& Borochowitz ZU 2012 A distinctive autosomal recessive syndrome of severe disproportionate short stature with short long bones, brachydactyly, and hypotrichosis in two consanguineous Arab families. European Journal of Medical Genetics 55 256-264. (doi:10.1016/j.ejmg.2012.02.011)

Silkworth WT, Nardi IK, Scholl LM \& Cimini D 2009 Multipolar spindle pole coalescence is a major source of kinetochore mis-attachment and chromosome mis-segregation in cancer cells. PLOS ONE 4 e6564. (doi:10.1371/journal.pone.0006564)
Stears A, O'Rahilly S, Semple RK \& Savage DB 2012 Metabolic insights from extreme human insulin resistance phenotypes. Best Practice \& Research. Clinical Endocrinology \& Metabolism 26 145-157. (doi:10.1016/j.beem. 2011.09.003)

Strnad P, Leidel S, Vinogradova T, Euteneuer U, Khodjakov A \& Gonczy P 2007 Regulated HsSAS-6 levels ensure formation of a single procentriole per centriole during the centrosome duplication cycle. Developmental Cell 13 203-213. (doi:10.1016/j.devcel.2007.07.004)

Venoux M, Tait X, Hames RS, Straatman KR, Woodland HR \& Fry AM 2013 Poc1A and Poc1B act together in human cells to ensure centriole integrity. Journal of Cell Science 126 163-175. (doi:10.1242/jcs. 111203)

Vitre BD \& Cleveland DW 2012 Centrosomes, chromosome instability (CIN) and aneuploidy. Current Opinion in Cell Biology 24 809-815. (doi:10.1016/j.ceb.2012.10.006)

Watanabe K, Kobayashi K, Takemoto M, Ishibashi R, Yamaga M, Kawamura H, Fujimoto M, Ishikawa T, Onishi S, Okabe E et al. 2013 Sitagliptin improves postprandial hyperglycemia by inhibiting glucagon secretion in Werner syndrome with diabetes. Diabetes Care 36 e119. (doi:10.2337/dc13-0709)

Willems M, Genevieve D, Borck G, Baumann C, Baujat G, Bieth E, Edery P, Farra C, Gerard M, Heron D et al. 2010 Molecular analysis of pericentrin gene (PCNT) in a series of 24 Seckel/microcephalic osteodysplastic primordial dwarfism type II (MOPD II) families. Journal of Medical Genetics 47 797-802. (doi:10.1136/jmg.2009.067298)

Received in final form 3 August 2015

Accepted 10 August 2015 http://jme.endocrinology-journals.org DOI: 10.1530/JME-15-0090 (c) 2015 The authors Printed in Great Britain
Published by Bioscientifica Ltd 